\title{
Ability of dynamic preload indices to predict fluid responsiveness in a high femoral-to-radial arterial pressure gradient: a retrospective study
}

\section{Seon Ju Kim', So Yeon Kim², Hye Sun Lee ${ }^{3}$, Goeun Park ${ }^{3}$, Eun Jang Yoon ${ }^{2}$, Sungtaik Heo', and Bon-Nyeo Koo ${ }^{2}$}

'Department of Anesthesiology and Pain Medicine, National Health Insurance Service Ilsan Hospital, Goyang, 'Department of Anesthesiology and Pain Medicine, Yonsei University College of Medicine, ${ }^{3}$ Department of Research Affairs, Biostatistics Collaboration Unit, Yonsei University College of Medicine, Seoul, Korea
Received December 31, 2020

Revised July 7, 2021

Accepted July 20, 2021

\section{Corresponding author}

Bon-Nyeo Koo, M.D., Ph.D.

Department of Anesthesiology and

Pain Medicine, Yonsei University

College of Medicine, 50-1 Yonsei-ro,

Seodaemun-gu, Seoul 03722, Korea

Tel: 82-2-2228-2420

Fax: 82-2-312-7185

E-mail: koobn@yuhs.ac
Background: Dynamic preload indices may predict fluid responsiveness in end-stage liver disease. However, their usefulness in patients with altered vascular compliance is uncertain. This study is the first to evaluate whether dynamic indices can reliably predict fluid responsiveness in patients undergoing liver transplantation with a high femoral-to-radial arterial pressure gradient (PG).

Methods: Eighty liver transplant recipients were retrospectively categorized as having a normal ( $n=56$ ) or high ( $n=24$, difference in systolic pressure $\geq 10 \mathrm{mmHg}$ and/or mean pressure $\geq 5 \mathrm{mmHg}$ ) femoral-to-radial arterial PG, measured immediately after radial and femoral arterial cannulation. The ability of dynamic preload indices (stroke volume variation, pulse pressure variation [PPV], pleth variability index) to predict fluid responsiveness was assessed before the surgery. Fluid replacement of $500 \mathrm{ml}$ of crystalloid solution was performed over $15 \mathrm{~min}$. Fluid responsiveness was defined as $\geq 15 \%$ increase in the stroke volume index. The area under the receiver-operating characteristic curve (AUC) indicated the prediction of fluid responsiveness.

Results: Fourteen patients in the normal, and eight in the high PG group were fluid responders. The AUCs for PPV in the normal, high PG groups and total patients were 0.702 (95\% confidence interval [Cl] 0.553-0.851, $\mathrm{P}=0.008), 0.633$ (95\% Cl 0.384-0.881, $\mathrm{P}=0.295$ ) and 0.667 (95\% Cl 0.537-0.798, $\mathrm{P}=0.012)$, respectively. No other index predicted fluid responsiveness.

Conclusions: PPV can be used as a dynamic index of fluid responsiveness in patients with end-stage liver disease but not in patients with altered vascular compliance.

Keywords: End stage liver disease; Fluid therapy; Hemodynamic monitoring; Liver transplantation.

\section{INTRODUCTION}

Patients with end-stage liver disease who undergo liver transplantation have hyperdynamic circulation with increased cardiac output (CO) and low systemic vascular resistance (SVR) [1]. Liver transplantation increases the risk of

This is an Open Access article distributed under the terms of the Creative Commons Attribution Non-Commercial License (http://creativecommons.org/licenses/by-nc/4.0) which permits unrestricted non-commercial use, distribution, and reproduction in any medium, provided the original work is properly cited.

Copyright (C) the Korean Society of Anesthesiologists, 2021 
massive bleeding and sudden hemodynamic changes during surgery [2]. Although fluid management plays an important role in maintaining blood pressure during liver transplantation, excessive fluid loading may worsen postoperative outcomes. In general, the goal of fluid therapy in hemodynamically unstable patients is to increase $\mathrm{CO}$ and optimize tissue perfusion [3,4].

Various indices have been used to predict individual responses to fluid therapy. It has been found that dynamic indices such as stroke volume variation (SVV) and pulse pressure variation (PPV) are more reliable than static indices such as central venous pressure (CVP) or pulmonary arterial occlusion pressure (PAOP) [5-7]. Several studies have shown that use of SVV or PPV is beneficial, especially in hemodynamically unstable patients in intensive care units and in patients undergoing cardiac surgery $[8,9]$. The pleth variability index (PVI) is also as accurate as SVV in predicting fluid responsiveness, and intraoperative fluid management based on the PVI has been shown to reduce intraoperative and postoperative lactate levels [10].

However, there is a lot of debate about the usefulness of dynamic indices as an indicator of need for intraoperative fluid therapy in patients with end-stage liver disease undergoing liver transplantation [11-14]. The predictability of dynamic indices may be affected by vascular compliance; SVV did not predict fluid responsiveness in patients with coronary artery disease who had a wide pulse pressure $(>60$ $\mathrm{mmHg}$ ) [15]. Differences in the femoral-to-radial arterial pressure are often observed during liver transplantation [16]. However, no study has determined the ability of dynamic indices to predict fluid responsiveness in this condition. The aim of this study was to evaluate whether or not dynamic indices can reliably predict fluid responsiveness in patients undergoing liver transplantation with a high femoral-to-radial arterial pressure gradient (PG).

\section{MATERIALS AND METHODS}

\section{Study population}

The study protocol was approved by the Institutional Review Board (IRB) of Severance Hospital, Yonsei University Health System (no. 4-2019-0034). Patient records and information were anonymized before analysis; hence, the requirement for written informed consent to access medical records was waived. We retrospectively identified 91 patients over 20 years of age who underwent elective living donor liv- er transplantation at our hospital between August 1, 2017, and January 31, 2019. Patients with preoperative arrhythmia, reduced left ventricular function (ejection fraction $<40 \%$ ), valvular heart disease, pulmonary hypertension, pulmonary disease (asthma, chronic obstructive pulmonary disease, history of lung resection), or chronic renal disease were excluded along with those who had incomplete medical records.

\section{Anesthesia and hemodynamic monitoring}

All patients underwent our institution's standard anesthesia protocol for living donor liver transplantation. Routine non-invasive monitoring (5-lead electrocardiography, pulse oximetry, non-invasive blood pressure, and the bispectral index) was applied. The Masimo Radical 7 co-oximeter probe (MasimoSET ${ }^{\circledR}$ Rainbow, Masimo Corp., USA) was placed on the patient's index finger and covered with a shield to eliminate light interference, as recommended by the manufacturer. Anesthesia was induced with intravenous propofol, sufentanil, and rocuronium and maintained with desflurane and a continuous infusion of sufentanil and rocuronium. After tracheal intubation, mechanical ventilation was started in volume-controlled mode with a tidal volume of $8 \mathrm{ml} / \mathrm{kg}$, a respiratory rate of $10-15$ breaths $/ \mathrm{min}$, and $5 \mathrm{~cm}$ $\mathrm{H}_{2} \mathrm{O}$ of positive end-expiratory pressure. Arterial catheters were inserted into the right radial artery and left femoral artery, and a Flotrac/Vigileo system (Edwards Lifesciences, USA) was connected to the radial arterial cannula. A pulmonary artery catheter (Swan-Ganz CCOmbo, Edwards Lifesciences) was inserted through the right internal jugular vein and connected to a Vigilance monitor (Edwards Lifesciences) for continuous measurement of CO. A central venous catheter was inserted in the left internal jugular vein or subclavian vein. All transducers were zeroed at the mid-axillary level. After induction of anesthesia and before the start of surgery, fluid loading was performed with $500 \mathrm{ml}$ of balanced crystalloid solution (Plasma Solution- $\mathrm{A}^{\circledR}$, CJ HealthCare, Korea) through a central line for $15 \mathrm{~min}$ following our institution's routine liver transplantation anesthesia protocol. This fluid loading was performed to prevent hypotension during anesthesia and to confirm the patient's fluid responsiveness. However, such crystalloid administration was excluded in patients with pre-existing lung disease, pulmonary hypertension, or chronic kidney disease, which was consistent with the exclusion criteria of the present study. A continuous infusion of norepinephrine was used to main- 
tain a mean arterial pressure (MAP) $>60 \mathrm{mmHg}$.

\section{Acquisition of demographic and hemodynamic data}

Preoperative characteristics, including age, sex, body mass index, left ventricular ejection fraction, and the model for end-stage liver disease score was collected from the electronic medical records. The following hemodynamic parameters were obtained from the anesthesia records: heart rate, radial arterial pressure, femoral arterial pressure, CVP, PAOP, PPV, PVI, CO, cardiac index, end-diastolic volume index (EDVI), right ventricular ejection fraction (RVEF), SVR, SVR index (SVRI), and stroke volume index (SVI). Each parameter was noted at the value $1 \mathrm{~min}$ before (baseline) and the value $5 \mathrm{~min}$ after the fluid loading was completed. CO, cardiac index, EDVI, RVEF, SVR, SVRI, and SVI were collected from the Swan-Ganz CCOmbo/Vigilance monitor, and SVV was obtained by the FloTrac/Vigileo system. The PPV and PVI were automatically measured using a patient monitor (Philips Intellivue, MX700, Philips, The Netherlands) and Masimo monitor with PVI software, respectively.

Patients were considered fluid responders if their SVI increased by at least 15\% (from Swan-GanzCCOmbo/Vigilance) after fluid loading $[17,18]$. Furthermore, the femoral-to-radial arterial PG was calculated to compare the effect of vascular tone on the ability of dynamic indices to predict fluid responsiveness. The difference between the femoral and the radial arterial blood pressures was measured immediately after radial and femoral arterial cannulation. A significant femoral-to-radial arterial PG was defined as a difference of $10 \mathrm{mmHg}$ in systolic arterial pressure (SAP) and/or 5 mmHg in MAP [19]. According to the femoral-to-radial arterial PG, patients were divided into the normal PG group (a difference in SAP $<10 \mathrm{mmHg}$ and $\mathrm{MAP}<5 \mathrm{mmHg}$ ) and the high $\mathrm{PG}$ group (a difference in SAP $\geq 10 \mathrm{mmHg}$ and/or MAP $\geq 5 \mathrm{mmHg}$ ).

\section{Statistical analysis}

To compare the demographic and hemodynamic data between the two groups (normal PG and high PG), continuous variables were analyzed using a Student's $t$-test and categorical variable was analyzed using a chi-square test. Continuous variables were examined for normality by the Kolmogorov-Smirnov test. Variables that did not deviated from normal distribution were presented as mean and standard devi- ation, and were compared using the independent sample $t$-test. Variables that were not normally distributed were presented as median and interquartile range, and were compared using the Mann-Whitney $U$ test. A paired $t$-test was used to compare hemodynamic variables of baseline and after fluid loading. The ability of the preload parameters (CVP, PAOP, SVV, PPV, and PVI) to predict fluid responsiveness was evaluated using receiver-operating characteristic (ROC) curves. Static preload parameters (CVP and PAOP) and dynamic preload parameters (SVV, PPV, and PVI) obtained 1 min before fluid loading were used to analyze ROC curves. The area under the ROC curve (AUC) of each value was calculated, and the respective values were compared with a value of 0.5. The comparison of AUC between the two groups of normal PG and high PG was analyzed using a z-test. The optimal cut-off values for preload variables were determined by considering the values that yielded the Youden index (Sensitivity + Specificity -1 ) for predicting fluid responsiveness. Statistical analyses were performed using SPSS (version 23, IBM Corp., USA) and SAS (version 9.4, SAS Inc., USA). A P value $<0.05$ was considered statistically significant.

\section{RESULTS}

Data for 80 of the 91 patients were available for the final analysis. Fifty-six of these 80 patients were in the normal PG group and 24 were in the high PG group. There was no significant between-group difference in the patient characteristics (Table 1).

Hemodynamic data of baseline and after fluid replacement are listed in Table 2. After fluid replacement, radial MAP, femoral MAP, CVP, PAOP, CO, cardiac index, and EDVI were significantly increased whereas SVV and PPV were significantly decreased in the normal PG group. In the high PG group, the CVP, PAOP, CO, and cardiac index were significantly increased and the SVV was significantly decreased after fluid replacement.

The baseline hemodynamic data for the fluid responders and non-responders in each group are listed in Table 3. Fourteen patients in the normal PG group and eight in the high PG group responded to fluid replacement. In the normal PG group, baseline SVR, SVRI, and PPV were higher in the responders, whereas $\mathrm{CO}, \mathrm{CI}$, and SVI were lower in the responders than those in the non-responders. However, there was no significant difference in the baseline hemodynamic data between responders and non-responders in the high PG group. 
Table 1. Patient Characteristics

\begin{tabular}{|c|c|c|c|}
\hline Variable & Normal PG $(n=56)$ & High PG $(n=24)$ & $P$ value \\
\hline Age (yr) & $54.8 \pm 9.2$ & $55.8 \pm 10.5$ & 0.671 \\
\hline Sex, male & $40(71.4)$ & $17(70.8)$ & 0.957 \\
\hline Height (cm) & $168(161,171.5)$ & $167(157.5,172)$ & $>0.999$ \\
\hline Weight (kg) & $69.2 \pm 11.6$ & $65.1 \pm 14.3$ & 0.185 \\
\hline BMl & $24.9 \pm 3$ & $23.7 \pm 3.7$ & 0.129 \\
\hline MELD & $10.79(7.92,14.58)$ & $12.52(8.98,19.48)$ & 0.156 \\
\hline $\operatorname{LVEF}(\%)$ & $68.7 \pm 5.6$ & $69.3 \pm 5.8$ & 0.686 \\
\hline Creatinine (mg/dl) & $0.74 \pm 0.27$ & $0.76 \pm 0.29$ & 0.696 \\
\hline
\end{tabular}

Values are expressed as the mean $\pm \mathrm{SD}$, number of patients (\%), or median (1Q, 3Q). BMI: body mass index, LVEF: left ventricular ejection fraction, MELD: model for end-stage liver disease, PG: pressure gradient.

Table 2. Hemodynamic Data of Before and After Fluid Replacement

\begin{tabular}{|c|c|c|c|c|c|c|}
\hline \multirow{2}{*}{ Variable } & \multicolumn{3}{|c|}{ Normal PG $(n=56)$} & \multicolumn{3}{|c|}{ High PG $(n=24)$} \\
\hline & Before & After & $P$ value & Before & After & $P$ value \\
\hline HR (beats/min) & $69 \pm 11$ & $77 \pm 16$ & $<0.001$ & $72 \pm 15$ & $78 \pm 15$ & 0.041 \\
\hline Radial MAP (mmHg) & $80 \pm 14$ & $91 \pm 18$ & 0.001 & $83 \pm 20$ & $88 \pm 15$ & 0.104 \\
\hline Femoral MAP (mmHg) & $82 \pm 14$ & $92 \pm 19$ & $<0.001$ & $89 \pm 20$ & $91 \pm 14$ & 0.596 \\
\hline CVP (mmHg) & $10 \pm 4$ & $12 \pm 5$ & $<0.001$ & $10 \pm 4$ & $12 \pm 4$ & 0.035 \\
\hline PAOP (mmHg) & $13 \pm 5$ & $16 \pm 6$ & $<0.001$ & $15 \pm 6$ & $19 \pm 6$ & 0.003 \\
\hline $\mathrm{CO}$ (L/min) & $6.5 \pm 2.4$ & $7.2 \pm 2$ & 0.008 & $6.1 \pm 2.3$ & $7.2 \pm 3$ & 0.002 \\
\hline $\mathrm{Cl}\left(\mathrm{L} / \mathrm{min} / \mathrm{m}^{2}\right)$ & $3.7 \pm 1$ & $4.1 \pm 1$ & 0.013 & $3.5 \pm 1$ & $4.1 \pm 1.5$ & 0.002 \\
\hline EDVI $\left(\mathrm{ml} / \mathrm{m}^{2}\right)$ & $153 \pm 40$ & $160 \pm 37$ & 0.027 & $139 \pm 36$ & $144 \pm 29$ & 0.469 \\
\hline RVEF (\%) & $36 \pm 10$ & $34 \pm 9$ & 0.059 & $35 \pm 11$ & $34 \pm 7$ & 0.844 \\
\hline SVR (dynes/sec/cm5) & $966 \pm 364$ & $1,005 \pm 388$ & 0.379 & $1,112 \pm 462$ & $1,027 \pm 392$ & 0.217 \\
\hline SVRI (dynes $/ \mathrm{sec} / \mathrm{cm}^{5} / \mathrm{m}^{2}$ ) & $1,713 \pm 705$ & $1,629 \pm 631$ & 0.346 & $1,860 \pm 745$ & $1,696 \pm 670$ & 0.163 \\
\hline SVI (ml/m²/beat) & $52 \pm 15$ & $52 \pm 13$ & 0.719 & $47 \pm 14$ & $50 \pm 15$ & 0.226 \\
\hline SWV (\%) & $7 \pm 3$ & $5 \pm 3$ & $<0.001$ & $6 \pm 3$ & $4 \pm 2$ & $<0.001$ \\
\hline PPV (\%) & $7 \pm 3$ & $5 \pm 2$ & $<0.001$ & $7 \pm 4$ & $5 \pm 2$ & 0.060 \\
\hline PVI (\%) & $11 \pm 5$ & $12 \pm 6$ & 0.276 & $10 \pm 6$ & $11 \pm 5$ & 0.723 \\
\hline
\end{tabular}

Values are expressed as the mean \pm SD. Cl: cardiac index, CO: cardiac output, CVP: central venous pressure, EDVI: end-diastolic volume index, HR: heart rate, MAP: mean arterial pressure, PAOP: pulmonary artery occlusive pressure, PG: pressure gradient, PPV: pulse pressure variation, PVI: pleth variability index, RVEF: right ventricular ejection fraction, SVI: stroke volume index, SVR: systemic vascular resistance, SVRI: systemic vascular resistance index, SWV: stroke volume variation.

The AUCs for the ability of several preload indices to predict an increase in SVI of $\geq 15 \%$ are shown in Fig. 1. The AUCs for PPV as a predictor of fluid responsiveness were 0.702 (95\% confidence interval [CI] 0.553-0.851, $\mathrm{P}=0.008$ ), 0.633 (95\% CI 0.384-0.881, P = 0.295) and 0.667 (95\% CI 0.537-0.798, $\mathrm{P}=0.012$ ) in the normal PG group, high PG group and whole cohort of patients, respectively. The threshold PPV value of $6 \%$ discriminated between responders and non-responders to fluid replacement with a sensitivity of $86 \%$ and a specificity of $45 \%$ in the normal PG group. There were no statistically significant between-group differences in the AUCs for CVP, PAOP, SVV, or PVI (Table 4).

\section{DISCUSSION}

In this study, we analyzed the ability of dynamic preload indices to predict fluid responsiveness in patients undergoing elective liver transplantation. We divided patients into two groups according to their femoral-to-radial arterial PG and investigated whether or not the predictive ability of these dynamic indices is affected by vascular compliance. Thirty percent of patients showed a significant femoral-to-radial arterial $\mathrm{PG}$ (difference in $\mathrm{SAP} \geq 10 \mathrm{mmHg}$ and/or MAP $\geq 5 \mathrm{mmHg}$ ). Only PPV was found to be a reliable predictor of fluid responsiveness in the patients with normal PGs, whereas no dynamic preload index appeared to be sensitive enough to predict fluid responsiveness in patients with high PGs. 
Table 3. Hemodynamic Data before Fluid Replacement in Fluid Responders and Non-responders

\begin{tabular}{|c|c|c|c|c|c|c|}
\hline \multirow[b]{2}{*}{ Variable } & \multicolumn{3}{|c|}{ Normal PG $(n=56)$} & \multicolumn{3}{|c|}{ High PG $(n=24)$} \\
\hline & $\begin{array}{l}\text { Responders } \\
\quad(n=14)\end{array}$ & $\begin{array}{c}\text { Non-responders } \\
(n=42)\end{array}$ & $P$ value & $\begin{array}{l}\text { Responders } \\
\quad(n=8)\end{array}$ & $\begin{array}{c}\text { Non-responders } \\
(n=16)\end{array}$ & $P$ value \\
\hline HR (beats/min) & $70 \pm 9$ & $69 \pm 12$ & 0.699 & $78 \pm 18$ & $69 \pm 13$ & 0.182 \\
\hline Radial MAP (mmHg) & $78 \pm 12$ & $81 \pm 15$ & 0.484 & $71 \pm 19$ & $88 \pm 19$ & 0.051 \\
\hline Femoral MAP (mmHg) & $80 \pm 12$ & $82 \pm 14$ & 0.609 & $79 \pm 17$ & $95 \pm 19$ & 0.059 \\
\hline CVP (mmHg) & $9 \pm 4$ & $10 \pm 4$ & 0.192 & $11 \pm 6$ & $10 \pm 4$ & 0.752 \\
\hline PAOP (mmHg) & $11 \pm 4$ & $14 \pm 5$ & 0.050 & $14 \pm 7$ & $16 \pm 6$ & 0.474 \\
\hline $\mathrm{CO}(\mathrm{L} / \mathrm{min})$ & $5.5 \pm 1.4$ & $6.9 \pm 2.5$ & 0.011 & $6.6 \pm 3.5$ & $5.9 \pm 1.5$ & 0.566 \\
\hline $\mathrm{Cl}\left(\mathrm{L} / \mathrm{min} / \mathrm{m}^{2}\right)$ & $3.2 \pm 0.7$ & $3.9 \pm 1.2$ & 0.035 & $3.7 \pm 1.5$ & $3.4 \pm 0.8$ & 0.574 \\
\hline EDVI $\left(\mathrm{ml} / \mathrm{m}^{2}\right)$ & $146 \pm 37$ & $156 \pm 41$ & 0.449 & $150 \pm 51$ & $134 \pm 27$ & 0.434 \\
\hline RVEF (\%) & $32 \pm 10$ & $37 \pm 9$ & 0.124 & $32 \pm 16$ & $36 \pm 8$ & 0.490 \\
\hline SVR (dynes/sec/cm5) & $1,158 \pm 414$ & $902 \pm 326$ & 0.021 & $961 \pm 530$ & $1,188 \pm 423$ & 0.265 \\
\hline SVRI (dynes/sec/cm $/ \mathrm{cm}^{2}$ ) & $2,212 \pm 856$ & $1,547 \pm 567$ & 0.015 & $1,564 \pm 635$ & $2,008 \pm 769$ & 0.174 \\
\hline SVI (ml/m²/beat) & $45 \pm 11$ & $55 \pm 15$ & 0.026 & $43 \pm 19$ & $49 \pm 10$ & 0.487 \\
\hline SWV (\%) & $8 \pm 3$ & $7 \pm 3$ & 0.242 & $6 \pm 2$ & $7 \pm 3$ & 0.395 \\
\hline PPV (\%) & $9 \pm 3$ & $7 \pm 3$ & 0.023 & $8 \pm 4$ & $6 \pm 3$ & 0.292 \\
\hline PVI (\%) & $11 \pm 6$ & $11 \pm 5$ & 0.752 & $12 \pm 9$ & $9 \pm 5$ & 0.503 \\
\hline
\end{tabular}

Values are expressed as the mean \pm SD. Cl: cardiac index, CO: cardiac output, CVP: central venous pressure, EDVI: end-diastolic volume index, HR: heart rate, MAP: mean arterial pressure, PAOP: pulmonary artery occlusive pressure, PG: pressure gradient, PPV: pulse pressure variation, PVI: pleth variability index, RVEF: right ventricular ejection fraction, SVI: stroke volume index, SVR: systemic vascular resistance, SVRI: systemic vascular resistance index, SVV: stroke volume variation.
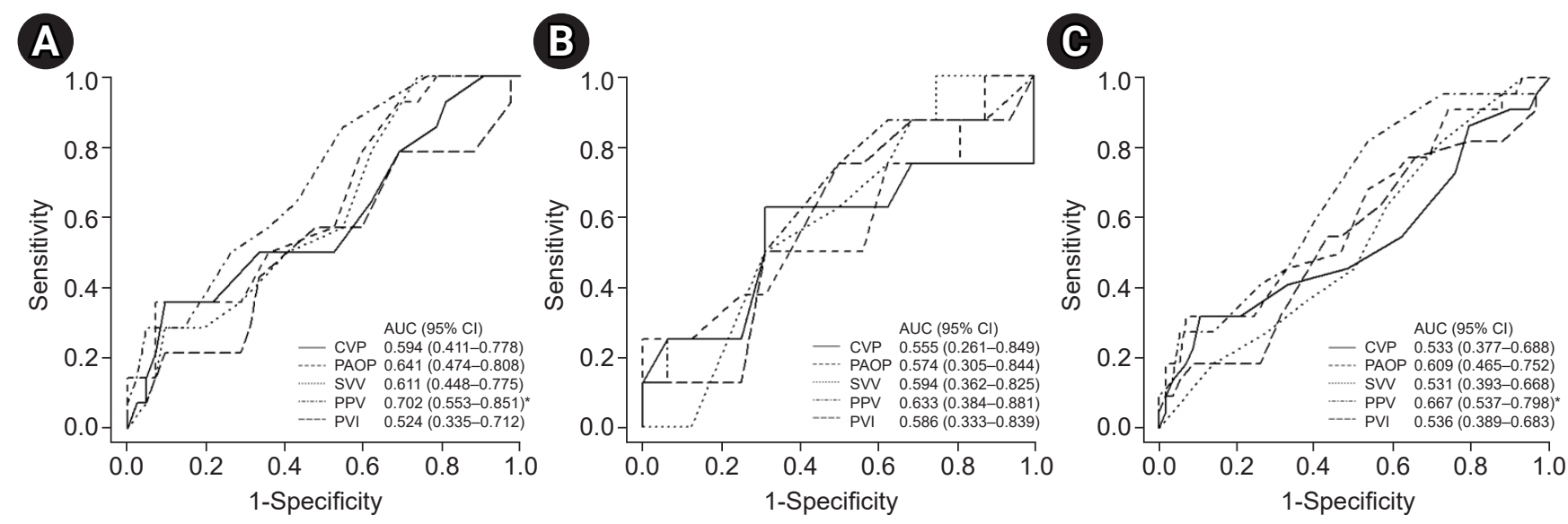

Fig. 1. Receiver-operating characteristic curves showing the ability of static and dynamic preload indices to predict an increase in stroke volume index $\geq 15 \%$ after fluid challenge. (A) Normal femoral-to-radial arterial pressure gradient group $(n=56)$. (B) High femoral-to-radial arterial pressure gradient group ( $n=24$, difference of $\geq 10 \mathrm{mmHg}$ in systolic blood pressure and/or mean blood pressure $\geq 5 \mathrm{mmHg}$ ). (C) Whole cohort of patients $(n=80)$. CVP: central venous pressure, PAOP: pulmonary arterial occlusion pressure, PPV: pulse pressure variation, $\mathrm{PVI}$ : pleth variability index, SVV: stroke volume variation. ${ }^{*} \mathrm{P}<0.05$.

Dynamic variables have been reported to be useful for predicting fluid responsiveness in patients with septic shock and those undergoing cardiac surgery with cardiopulmonary bypass who may have a similar physiology to that of patients with end-stage liver disease $[9,20,21]$. However, previous studies of fluid responsiveness in liver transplant patients have not yielded consistent results [11-14]. In a study of 31 patients who received liver transplantation with 62 fluid challenges, dynamic indices of SVV, PPV, and PVI were useful for predicting fluid responsiveness with AUCs of 0.754, 0.794, and 0.800 , respectively [11]. Moreover, the femoral SVV obtained using the Vigileo monitor could reflect fluid responsiveness with an AUC of 0.894 in recipients during the anhepatic phase of liver transplantation [12]. However, the PPV 
Table 4. Comparisons of Area Under the Receiver-operating Characteristic Curve between Normal PG Group and High PG Group

\begin{tabular}{|c|c|c|c|c|c|}
\hline \multirow{2}{*}{ Preload indices } & \multicolumn{2}{|c|}{ Normal PG $(n=56)$} & \multicolumn{2}{|c|}{ High PG $(n=24)$} & \multirow{2}{*}{$P$ value } \\
\hline & Area under the curve & Standard error & Area under the curve & Standard error & \\
\hline CVP (mmHg) & 0.594 & 0.094 & 0.555 & 0.150 & 0.822 \\
\hline PAOP (mmHg) & 0.641 & 0.085 & 0.574 & 0.138 & 0.679 \\
\hline SWV (\%) & 0.611 & 0.083 & 0.594 & 0.118 & 0.903 \\
\hline PPV (\%) & 0.702 & 0.076 & 0.633 & 0.127 & 0.638 \\
\hline PVI (\%) & 0.524 & 0.096 & 0.586 & 0.129 & 0.700 \\
\hline
\end{tabular}

CVP: central venous pressure, PAOP: pulmonary artery occlusive pressure, PG: pressure gradient, PPV: pulse pressure variation, PVI: pleth variability index, SVV: stroke volume variation.

failed to predict fluid responsiveness during liver transplantation in another study [13]. Likewise, Konur et al. [14] reported that the PVI derived from a Masimo monitor was not a reliable predictor of fluid responsiveness during the dissection phase or anhepatic phase in liver transplantation. In the present study, PPV was a reliable predictor of fluid responsiveness with an AUC of 0.702 in patients with a normal PG, but failed to predict fluid responsiveness in those with a high PG. In contrast, SVV and PVI could not predict fluid responsiveness in patients with a normal PG or those with a high PG.

It is not known exactly why the predictive ability of dynamic indices varies in patients undergoing liver transplantation, but it may be due to the characteristic physiology of patients with end-stage liver disease. Patients with liver cirrhosis often present with hyperdynamic circulation characterized by an increased heart rate and CO with reduced SVR $[1,22]$. Splanchnic arterial vasodilation in cirrhosis leads to a functional hypovolemia (decreased preload) despite a volume overload in absolute terms [23]. Even with an increased basal CO, patients with cirrhosis show blunted responsiveness to volume, exercise, or pharmacological stimuli, known as cirrhotic cardiomyopathy [23]. This blunted cardiac response of cirrhotic cardiomyopathy fails to overcome the decrease in the effective circulating volume [23]. Owing to these characteristics of end-stage liver disease, the dynamic indices, which predict fluid responsiveness well under other conditions, may fail to predict fluid responsiveness in patients undergoing liver transplantation.

There are several confounders in clinical practice that can significantly reduce the predictive value of dynamic indices of fluid responsiveness, including low tidal volume, cardiac arrhythmias, intra-abdominal hypertension, elevated positive end-expiratory pressure, and use of vasopressor drugs $[24,25]$. In particular, the SVV failed to predict fluid responsiveness in patients with increased arterial stiffness [15]. Moreover, changes in vascular tone with a vasoconstrictor or vasodilator can affect the predictability of the dynamic index
$[26,27]$. An animal study reported that the predictability of PPV or systolic pressure variation in fluid responsiveness is poor when vasomotor tone is increased by infusion of the al agonist phenylephrine [26]. In addition, vasodilator treatment in patients ventilated postoperatively created a relative hypovolemic state, resulting in an increased PPV and SVV [27], which may affect the reliability of these indices.

In the current study, we divided patients into two groups according to their femoral-to-radial arterial PG and investigated whether or not the predictability of dynamic variables is affected by vascular compliance. An arterial pressure difference between the femoral artery and the radial artery is often observed during liver transplantation [28]. This is similar to that observed after cardiopulmonary bypass in cardiac surgery or deep hypothermic circulatory arrest and is often caused by peripheral arterial vasodilation $[29,30]$. Therefore, the high PG in our patients may reflect severe peripheral arterial vasodilation. [16] Our study is the first to demonstrate that dynamic indices such as PPV, SVV, and PVI cannot be used to predict fluid responsiveness in the condition of a high femoral-to-radial arterial PG.

There are several limitations to our study. First, the data were collected retrospectively, which introduces potential confounding factors. However, the anesthesia of all the enrolled patients was performed by one anesthesiologist, and the data were entered into the electronic medical records according to our protocol. Moreover, the number of patients analyzed in our study was larger $(n=80)$ than that in previous studies investigating fluid responsiveness in liver transplant patients ( $\mathrm{n}=15-37$ ) [11-14]; this may help overcome the limitation of the retrospective design. Second, our patients received $500 \mathrm{ml}$ of crystalloid solution based on our institution's routine anesthesia protocol, which is less than that in previous studies $(10 \mathrm{ml} / \mathrm{kg}$ of colloids or crystalloid solution) on liver transplantation $[11,12,14]$. Therefore, the $500 \mathrm{ml}$ of crystalloid administered in our study could have been insufficient to assess fluid responsiveness and may 
have affected the predictability of dynamic indices in the patients with normal PG. Third, use of a vasoconstrictor such as norepinephrine can increase vascular tone [26], which could affect the predictive ability of dynamic indices. Given the retrospective design of our study, the contribution of norepinephrine could not be ascertained. Fourth, in our study, the radial arterial catheter was connected to the Flotrac/Vigileo monitor, and the femoral arterial catheter was connected to a disposable pressure transducer (Edwards Lifesciences). According to the manufacturer, the proprietary algorithm of the Flotrac/Vigileo system allows CO monitoring independent of the signal detection site, considering the differences in the vascular structure. Additionally, the two transducers were simultaneously calibrated at the mid axillary level for atmospheric pressure (zeroed) in the present study. Therefore, although arterial pressure was not obtained via the radial and femoral arterial catheters using the same transducer, the difference in the arterial pressure is considered to be insignificant. Finally, our data were collected after induction of anesthesia and before the start of surgery. Therefore, the results of the study did not reflect the overall state of liver transplantation. Liver transplantation has different hemodynamic changes in each phase; therefore, further studies are required to identify reliable predictors in each phase.

In conclusion, patients with end-stage liver disease undergoing liver transplantation have extreme systemic arterial vasodilation, and this phenomenon could affect the reliability of the dynamic preload indices that have been widely used to predict fluid responsiveness. Our study demonstrated that PPV can be used as a dynamic index of fluid responsiveness in patients with end-stage liver disease. However, caution is needed when using the PPV to guide fluid therapy in patients with altered vascular compliance, such as a high femoral-to-radial arterial PG. Further studies are needed to identify reliable predictors of fluid responsiveness in patients with altered vascular compliance.

\section{CONFLICTS OF INTEREST}

No potential conflict of interest relevant to this article was reported.

\section{DATA AVAILABILITY STATEMENT}

The datasets generated during and/or analyzed during the current study are available from the corresponding author on reasonable request.

\section{AUTHOR CONTRIBUTIONS}

Conceptualization: Seon Ju Kim, Bon-Nyeo Koo. Data curation: Seon Ju Kim, Sungtaik Heo. Formal analysis: Hye Sun Lee, Goeun Park. Methodology: So Yeon Kim, Bon-Nyeo Koo. Project administration: Bon-Nyeo Koo. Visualization: So Yeon Kim, Hye Sun Lee. Writing - original draft: Seon Ju Kim. Writing - review \& editing: So Yeon Kim, Bon-Nyeo Koo. Investigation: Seon Ju Kim, So Yeon Kim, Bon-Nyeo Koo, Eun Jang Yoon, Sungtaik Heo. Resources: Bon-Nyeo Koo. Supervision: Bon-Nyeo Koo. Validation: Seon Ju Kim, So Yeon Kim, Bon-Nyeo Koo.

\section{ORCID}

Seon Ju Kim, https://orcid.org/0000-0002-1911-8143

So Yeon Kim, https://orcid.org/0000-0001-5352-157X

Hye Sun Lee, https://orcid.org/0000-0001-6328-6948

Goeun Park, https://orcid.org/0000-0002-6670-5500

Eun Jang Yoon, https://orcid.org/0000-0002-2092-6658

Sungtaik Heo, https://orcid.org/0000-0002-8938-6119

Bon-Nyeo Koo, https://orcid.org/0000-0002-3189-1673

\section{REFERENCES}

1. Izzy M, VanWagner LB, Lin G, Altieri M, Findlay JY, Oh JK, et al. Redefining cirrhotic cardiomyopathy for the modern era. Hepatology 2020; 71: 334-45.

2. Schroeder RA, Collins BH, Tuttle-Newhall E, Robertson K, Plotkin J, Johnson LB, et al. Intraoperative fluid management during orthotopic liver transplantation. J Cardiothorac Vasc Anesth 2004; 18: 438-41.

3. Gan TJ, Soppitt A, Maroof M, el-Moalem H, Robertson KM, Moretti E, et al. Goal-directed intraoperative fluid administration reduces length of hospital stay after major surgery. Anesthesiology 2002; 97: 820-6.

4. Grocott MP, Mythen MG, Gan TJ. Perioperative fluid management and clinical outcomes in adults. Anesth Analg 2005; 100: 1093-106.

5. Osman D, Ridel C, Ray P, Monnet X, Anguel N, Richard C, et al. Cardiac filling pressures are not appropriate to predict hemodynamic response to volume challenge. Crit Care Med 2007; 35: 64-8.

6. Marik PE, Cavallazzi R, Vasu T, Hirani A. Dynamic changes in arterial waveform derived variables and fluid responsiveness 
in mechanically ventilated patients: a systematic review of the literature. Crit Care Med 2009; 37: 2642-7.

7. Preisman S, Kogan S, Berkenstadt H, Perel A. Predicting fluid responsiveness in patients undergoing cardiac surgery: functional haemodynamic parameters including the Respiratory Systolic Variation Test and static preload indicators. Br J Anaesth 2005; 95: 746-55.

8. Michard F, Teboul JL. Predicting fluid responsiveness in ICU patients: a critical analysis of the evidence. Chest 2002; 121: 2000-8.

9. Rex S, Brose S, Metzelder S, Hüneke R, Schälte G, Autschbach $\mathrm{R}$, et al. Prediction of fluid responsiveness in patients during cardiac surgery. Br J Anaesth 2004; 93: 782-8.

10. Forget P, Lois F, de Kock M. Goal-directed fluid management based on the pulse oximeter-derived pleth variability index reduces lactate levels and improves fluid management. Anesth Analg 2010; 111: 910-4.

11. Wu CY, Cheng YJ, Liu YJ, Wu TT, Chien CT, Chan KC. Predicting stroke volume and arterial pressure fluid responsiveness in liver cirrhosis patients using dynamic preload variables: a prospective study of diagnostic accuracy. Eur J Anaesthesiol 2016; 33: $645-52$.

12. Shin YH, Ko JS, Gwak MS, Kim GS, Lee JH, Lee SK. Utility of uncalibrated femoral stroke volume variation as a predictor of fluid responsiveness during the anhepatic phase of liver transplantation. Liver Transpl 2011; 17: 53-9.

13. Gouvêa G, Diaz R, Auler L, Toledo R, Martinho JM. Evaluation of the pulse pressure variation index as a predictor of fluid responsiveness during orthotopic liver transplantation. Br J Anaesth 2009 ; 103: 238-43.

14. Konur H, Erdogan Kayhan G, Toprak HI, Bucak N, Aydogan MS, Yologlu S, et al. Evaluation of pleth variability index as a predictor of fluid responsiveness during orthotopic liver transplantation. Kaohsiung J Med Sci 2016; 32: 373-80.

15. Kim SY, Song Y, Shim JK, Kwak YL. Effect of pulse pressure on the predictability of stroke volume variation for fluid responsiveness in patients with coronary disease. J Crit Care 2013; 28: 318.e1-e7.

16. Arnal D, Garutti I, Perez-Peña J, Olmedilla L, Tzenkov IG. Radial to femoral arterial blood pressure differences during liver transplantation. Anaesthesia 2005; 60: 766-71.

17. Reuter DA, Felbinger TW, Schmidt C, Kilger E, Goedje O, Lamm P, et al. Stroke volume variations for assessment of cardiac responsiveness to volume loading in mechanically ventilated patients after cardiac surgery. Intensive Care Med 2002; 28: 392-8.
18. Zimmermann M, Feibicke T, Keyl C, Prasser C, Moritz S, Graf $\mathrm{BM}$, et al. Accuracy of stroke volume variation compared with pleth variability index to predict fluid responsiveness in mechanically ventilated patients undergoing major surgery. Eur J Anaesthesiol 2010; 27: 555-61.

19. Hong SW, Shim JK, Choi YS, Chun DH, Kim JC, Kim BS, et al. Predictors of ineffectual radial arterial pressure monitoring in valvular heart surgery. J Heart Valve Dis 2009; 18: 546-53.

20. Khwannimit B, Bhurayanontachai R. Prediction of fluid responsiveness in septic shock patients: comparing stroke volume variation by FloTrac/Vigileo and automated pulse pressure variation. Eur J Anaesthesiol 2012; 29: 64-9.

21. Freitas FG, Bafi AT, Nascente AP, Assunção M, Mazza B, Azevedo LC, et al. Predictive value of pulse pressure variation for fluid responsiveness in septic patients using lung-protective ventilation strategies. Br J Anaesth 2013; 110: 402-8.

22. Kwon HM, Hwang GS. Cardiovascular dysfunction and liver transplantation. Korean J Anesthesiol 2018; 71: 85-91.

23. Ruiz-del-Árbol L, Serradilla R. Cirrhotic cardiomyopathy. World J Gastroenterol 2015; 21: 11502-21.

24. Lansdorp B, Lemson J, van Putten MJ, de Keijzer A, van der Hoeven JG, Pickkers P. Dynamic indices do not predict volume responsiveness in routine clinical practice. Br J Anaesth 2012; 108: 395-401.

25. Renner J, Scholz J, Bein B. Monitoring fluid therapy. Best Pract Res Clin Anaesthesiol 2009; 23: 159-71.

26. Bouchacourt JP, Riva JA, Grignola JC. The increase of vasomotor tone avoids the ability of the dynamic preload indicators to estimate fluid responsiveness. BMC Anesthesiol 2013; 13: 41.

27. Hadian M, Severyn DA, Pinsky MR. The effects of vasoactive drugs on pulse pressure and stroke volume variation in postoperative ventilated patients. J Crit Care 2011; 26: 328.e1-e8.

28. Lee M, Weinberg L, Pearce B, Scurrah N, Story DA, Pillai P, et al. Agreement between radial and femoral arterial blood pressure measurements during orthotopic liver transplantation. Crit Care Resusc 2015; 17: 101-7.

29. Chauhan S, Saxena N, Mehrotra S, Rao BH, Sahu M. Femoral artery pressures are more reliable than radial artery pressures on initiation of cardiopulmonary bypass. J Cardiothorac Vasc Anesth 2000; 14: 274-6.

30. Manecke GR Jr, Parimucha M, Stratmann G, Wilson WC, Roth DM, Auger WR, et al. Deep hypothermic circulatory arrest and the femoral-to-radial arterial pressure gradient. J Cardiothorac Vasc Anesth 2004; 18: 175-9. 